$4-2012$

\title{
Multivitamin Therapy for Recurrent Aphthous Stomatitis
}

\author{
Rajesh V. Lalla \\ University of Connecticut School of Medicine and Dentistry \\ Linda E. Choquette \\ University of Connecticut School of Medicine and Dentistry \\ Richard S. Feinn \\ University of Connecticut School of Medicine and Dentistry \\ Harriet Zawistowski \\ University of Connecticut School of Medicine and Dentistry \\ Marie C. Latortue \\ University of Connecticut School of Medicine and Dentistry \\ See next page for additional authors
}

Follow this and additional works at: https://opencommons.uconn.edu/uchcres_articles Part of the Dentistry Commons

\section{Recommended Citation}

Lalla, Rajesh V.; Choquette, Linda E.; Feinn, Richard S.; Zawistowski, Harriet; Latortue, Marie C.; and Kelly, Edward T., "Multivitamin Therapy for Recurrent Aphthous Stomatitis" (2012). UCHC Articles - Research. 214.

https://opencommons.uconn.edu/uchcres_articles/214 
Authors

Rajesh V. Lalla, Linda E. Choquette, Richard S. Feinn, Harriet Zawistowski, Marie C. Latortue, and Edward T. Kelly 
Published in final edited form as:

J Am Dent Assoc. 2012 April ; 143(4): 370-376.

\title{
Multivitamin therapy for recurrent aphthous stomatitis:
}

\author{
A randomized, double-masked, placebo-controlled trial
}

Dr. Rajesh V. Lalla, DDS, PhD, CCRP, DABOM,

Assistant professor, Section of Oral Medicine, University of Connecticut Health Center, MC1605,

263 Farmington Ave., Farmington, Conn. 06030-1605

Ms. Linda E. Choquette, RDH, MSHS, CCRP,

Clinical research associate, Section of Oral Medicine, University of Connecticut Health Center, Farmington

Dr. Richard S. Feinn, PhD,

Biostatistician, Clinical Research Center, University of Connecticut Health Center, Farmington

Ms. Harriet Zawistowski, BGS, CCRP,

Clinical research assistant, Clinical Research Center, University of Connecticut Health Center, Farmington

Dr. Marie C. Latortue, DDS, MPH, MS,

At the time this study was conducted, Dr. Latortue was a postdoctoral fellow in clinical research, Section of Oral Medicine, University of Connecticut Health Center, Farmington. She now is a resident in prosthodontics, New York University, New York City

Dr. Edward T. Kelly, DMD, and

At the time this study was conducted, Dr. Kelly was a dental student, School of Dental Medicine, University of Connecticut Health Center, Farmington. He now is in private practice

Dr. Lorena Baccaglini, DDS, MS, PhD, CCRP, DABOM

Assistant professor, College of Dentistry, University of Florida, Gainesville

\section{Abstract}

Background-Recurrent aphthous stomatitis (RAS) is a painful condition of unknown etiology, affecting more than 2.5 billion people worldwide. Vitamin deficiencies have been implicated as a possible cause.

Methods-The authors conducted a single-center, randomized, parallel-arm, double-masked, placebo-controlled study to examine the effect of daily multivitamin supplementation on the number and duration of RAS episodes. The authors randomly assigned 160 adults who had a validated history of at least three episodes of idiopathic minor RAS within the previous 12 months to one of two groups: the first group $(n=83)$ received a once-daily multivitamin containing 100 percent of the U.S. reference daily intake (RDI) of essential vitamins, and the second group ( $\mathrm{n}=$ 77) received once-daily placebo for up to 365 days.

Results-The results showed no significant difference in the mean number of new RAS episodes between the multivitamin (4.19 episodes) and placebo (4.60 episodes) arms during the study period $(P=.69)$. The mean duration of new RAS episodes also was similar for the multivitamin (8.66 days) and placebo ( 8.99 days) arms $(P=.60)$. Furthermore, the authors found no differences

Copyright @ 2012 American Dental Association. All rights reserved.

Address reprint requests to Dr. Lalla., lalla@uchc.edu.

Disclosure. None of the authors reported any disclosures. 
between the two arms with regard to mouth pain, normalcy of diet or compliance with the study medication regimen.

Conclusion-Daily multivitamin supplementation, with the RDI of essential vitamins, did not result in a reduction in the number or duration of RAS episodes.

Clinical Implications-Clinicians should not recommend multi-vitamin supplementation routinely as prophylaxis for RAS.

\section{Keywords}

Recurrent aphthous stomatitis; canker sore; multi-vitamin; vitamin

Recurrent aphthous stomatitis (RAS), also known as "canker sores," is a common disease, affecting an estimated 2.5 billion people worldwide. ${ }^{1}$ Clinically, this disease manifests as recurrent, painful oral ulcerations. Three clinical types have been described: minor, major and herpetiform. ${ }^{2}$ Owing to their painful nature, RAS lesions can affect nutrition, speech and oral hygiene. ${ }^{3}$ Thus, RAS can result in significant morbidity and a diminished quality of life. ${ }^{4,5}$ Patients who experience major RAS develop large, painful oral ulcerations that often last for several weeks and heal with scarring. The etiology of RAS is not well understood, which hampers the development of effective therapies for prevention or treatment. Several factors have been implicated, including genetics, stress, allergies and autoimmunity. ${ }^{2}$ In addition, a substantial body of literature indicates that some patients with RAS have vitamin deficiencies.

Researchers in several studies have implicated vitamin deficiencies—including vitamin $\mathrm{B}_{12},{ }^{6-10}$ folic acid, ${ }^{8,9,11}$ vitamin $\mathrm{B}_{1}, \mathrm{~B}_{2}$ and $\mathrm{B}_{6}$-in the pathogenesis of RAS. ${ }^{12,13}$ For example, investigators in one study found that 28 percent of patients with RAS had a deficiency of one or more of the $\mathrm{B}$ vitamins tested $\left(\mathrm{B}_{1}, \mathrm{~B}_{2}\right.$ and $\left.\mathrm{B}_{6}\right)$. In these patients, replacement therapy for the deficient vitamins significantly reduced the number of days with RAS. ${ }^{13}$ Investigators in other studies also reported that specific replacement therapy to correct vitamin deficiencies (notably $\mathrm{B}_{12}$ and folic acid) can be effective in improving the patient's condition or leading to remission. $., 10,14$ The results of studies of therapy with a combination vitamin/herbal supplement (LongoVital, Cederroth, Upplands Väsby, Sweden) indicated that daily prophylactic use resulted in a reduced number of RAS lesions, even in the absence of serologic vitamin deficiency. ${ }^{15,16}$ However, because this supplement contains both vitamins and herbal supplements, the specific contribution of vitamins is not clear.

Thus, the role of vitamin deficiencies in RAS is not well understood. Furthermore, testing for vitamin deficiencies is invasive and expensive, and it is not done routinely in the clinical management of RAS. Currently used therapies (such as steroids) have significant adverse effects. Therefore, we sought to identify a low-cost, non-toxic strategy for the prevention of RAS lesions. We performed this study to examine the efficacy of daily multivitamin supplementation in a group of patients with RAS.

\section{METHODS}

\section{Study design}

We conducted a randomized, double-masked, placebo-controlled, parallel-arm, single-center study at the University of Connecticut Health Center, Farmington, after having received institutional review board approval. All participants provided written informed consent. 


\section{Participants}

We recruited participants from the general population of Hartford County, Connecticut, via flyers posted at various locations, e-mails to the health center community and advertisements in local newspapers. People 18 years and older with a validated history of at least three episodes of idiopathic minor RAS within the previous 12 months were eligible for the study. One of three study investigators (R.V.L., L.E.C., H.Z.) questioned volunteers about the location, duration and appearance of reported lesions and showed them photographs of RAS lesions and of other oral ulcerative lesions to validate their history of RAS. Exclusion criteria included smoking, pregnancy, regular use of vitamin supplements or any over-the-counter or prescription agents for RAS, and a diagnosis of a systemic condition that can cause oral ulceration. Detailed exclusion criteria are available at www.clinicaltrials.gov, where the trial is registered under identifier number NCT00527306.

\section{Interventions}

The active agent tested was a generic multivitamin supplement containing only the U.S. reference daily intake (RDI) of the essential vitamins $A, B_{1}, B_{2}, B_{3}, B_{5}, B_{6}, B_{9}, B_{12}, C, D$ and $\mathrm{E} .{ }^{17}$ The active agent did not contain any other micronutrients or supplements such as iron or calcium. The placebo consisted of lactose powder. A pharmacy technician inserted the multivitamin and placebo into identically colored gelatin capsules at the University of Connecticut Health Center Research Pharmacy. Thus, the multivitamin and placebo capsules had the same external physical appearance, taste and texture. We instructed participants to take one capsule per day for 365 days and to record use of the medication in a study diary.

\section{Randomization and masking}

The research pharmacist assigned participants randomly to the multivitamin or placebo groups (1:1 allocation ratio) by using a computer-based pseudorandom number generator. We used a stratified randomization with a fixed block size of 20 participants. We defined strata as low RAS frequency (three or four RAS episodes in the year before the study; group A) or high RAS frequency (five or more episodes in the previous year; group B). The research pharmacist dispensed the treatments and kept confidential records of study drug assignment, which were not accessible to study investigators during the study.

\section{Clinical data collection}

Baseline visit-At the baseline study visit, we performed the following procedures: screened prospective participants; obtained medical histories, including use of any medications; conducted clinical oral examinations; educated participants about RAS and other oral ulcers; dispensed the study medication; distributed the study diary forms and instructions for study participation, including use of the study medication; and took a baseline blood sample to determine vitamin $\mathrm{B}_{12}$ and folate levels. We also showed photographs of RAS and non-RAS oral ulcers to participants for reference.

Six-month visit-At the six-month recall visit, one of three investigators (R.V.L., L.E.C., H.Z.) updated participants' medical histories, including current medications; performed a clinical oral examination; reeducated participants about RAS and other oral ulcers; dispensed additional study medication and study diary pages; collected completed study diary pages (from the first six months); and provided oral and written reinforcement of instructions in use of the study medication.

One-year visit-At the one-year study visit (the final visit), one of the three investigators performed the following procedures: collected completed study diary pages (from the 
second six months); updated medical histories, including current medications; and conducted clinical oral examinations.

In addition to the three scheduled study visits, we asked participants to return for additional study visits when they developed new episodes of mouth sores during the one-year study period. We defined a new episode as the onset of mouth sores after at least one day without any mouth sores. At these visits, a masked dentist or dental hygienist using calibrated technique obtained a detailed medical history and performed an oral examination to determine whether the mouth sores were RAS. If the dentist or dental hygienist determined that the mouth sores were not RAS, he or she referred the participant for additional clinical evaluation if appropriate. We paid participants $\$ 25$ for each completed study visit.

Participants completed written diaries consisting of data regarding onset and duration of each RAS episode experienced during study participation. We instructed them to record these data on the basis of the first and last day on which they were aware of the presence of an RAS lesion. In addition, participants recorded mouth pain scores and normalcy of diet scores for each day of an RAS episode. We gave participants postage-paid envelopes for return of the completed forms.

We asked participants to bring in bottles of capsules at the six- and 12-month study visits so that we could count the number of remaining pills. However, their compliance with this request was suboptimal and, therefore, we were unable to obtain satisfactory data regarding pill counts. In addition, at each study visit (that is, baseline, six months and any intervening RAS episode visit), we asked participants about compliance with the study medication regimen and reinforced the importance of taking the medication as instructed.

\section{Outcome measures}

The primary outcome measures were the number and duration of RAS episodes during the one-year study period. Secondary outcome measures included mouth pain and normalcy of diet during RAS episodes. We assessed mouth pain during RAS episodes by asking participants to indicate the daily level of mouth pain (worst, least, average and pain now) on an 11-point validated scale ranging from 0 (no pain) to 10 (worst pain imaginable). ${ }^{18} \mathrm{We}$ assessed normalcy of diet by using a validated Normalcy of Diet scale, ${ }^{19}$ on which participants rated (during RAS episodes) the highest ranking food they could eat each day (ranging from 0 [no alimentation possible] to 10 [full diet]).

\section{Sample size and statistical methods}

We calculated study sample size on the basis of 80 percent power and 0.05 a to detect at least a 65 percent probability that a randomly chosen participant from the multivitamin arm had a superior outcome (that is, fewer episodes, episodes of shorter duration or both) when compared with that for a randomly chosen participant from the placebo arm. On the basis of these parameters, we estimated that a final sample size of at least 100 participants (50 per arm) completing the study would be needed to achieve adequate power after accounting for dropouts.

We conducted baseline group comparisons of demographic variables and baseline vitamin levels by using the unpaired $t$ test for continuous measures and the $\chi^{2}$ test for categorical measures. A few participants with severe disease had multiple overlapping RAS lesions that they reported as one long episode. On the basis of published data ${ }^{20}$ indicating that the maximum duration of minor RAS episodes is 21 days, we treated such episodes as multiple episodes of up to 21 days each for the analyses. 
Main outcome measures-For the first main outcome-duration of RAS episodes-we used a linear mixed model with "treatment" (multivitamin or placebo) as the exposure and "duration in days" as the dependent variable. Episodes were clustered within participants and we treated the intercept as random, allowing for differing numbers of episodes among study participants. For the second main outcome-number of episodes-we used an analysis of covariance with "treatment" as the exposure and "length of study participation" as the covariate to enable us to adjust for a variable length of follow-up.

Secondary outcome measures-Analyses for the secondary outcome measuresmouth pain and diet—also involved the use of linear mixed models because participants could provide multiple observations for both of these measures. We included additional covariates - sex and number of episodes in the previous year-in the above models to assess their effects on parameter estimates. We did not perform any statistical analyses before all participants completed their participation in the study. We performed all analyses by using statistical software (PASW Statistics 18, IBM SPSS, Armonk, N.Y.).

\section{RESULTS}

\section{Participants}

We enrolled a total of 160 participants in this study and randomly assigned 83 to the multivitamin arm and 77 to the placebo arm. The stratified block randomization resulted in an even distribution of participants across the low and high RAS frequency groups (that is, groups A and B), as well as comparable demographics and baseline levels of vitamin $\mathrm{B}_{12}$ and red blood cell (RBC) folate across the two treatment arms (Table 1). Baseline vitamin $\mathrm{B}_{12}$ levels were low (less than 201 picograms per milliliters) in 14 of the 160 participants (9 percent). Of these 14 participants, five were randomized to the multivitamin arm (all five in group B) and nine were randomized to the placebo arm (four in group A [low RAS frequency] and five in group B [high RAS frequency]). For participants with low baseline levels of $B_{12}$, there was no significant difference in the incidence of new RAS episodes during the period of study participation between the five participants randomized to the multivitamin arm and the nine participants randomized to the placebo arm $(P=.0162)$. Baseline RBC folate levels were low (less than 237 nanograms $/ \mathrm{mL}$ ) in two participants. We randomly assigned one of these participants to the multivitamin arm (group B) and one to the placebo arm (group B). We did not perform any statistical analyses pertaining to low baseline RBC folate levels because of the low number of such participants.

Twenty-five of 83 participants ( 30 percent) in the multivitamin arm and 21 of 77 participants (27 percent) in the placebo arm did not complete the study. In most cases, they gave no specific reason for dropping out. Thus, 58 participants in the multivitamin arm and 56 participants in the placebo arm completed the entire one-year study. All participants (including those who withdrew) reported a total of 779 new RAS episodes during the study. For 575 of these episodes, the participant underwent a clinical examination during the episode; a dentist or dental hygienist confirmed that 498 of these 575 episodes (87 percent) were RAS and determined that 77 of these episodes (13 percent) were not RAS. We removed the latter episodes from the analyses.

\section{Compliance with study medication regimen}

We calculated compliance in two ways. When we compared the number of days that participants reported having taken their study medication with the total number of days for which they provided responses in the study diary, the mean compliance rates were 92.6 percent and 94.4 percent for the multivitamin and placebo arms, respectively $(P=.43)$. However, when we compared the number of days that participants reported taking the study 
medication with the total number of days that they were in the study (thus, assuming conservatively that participants did not take the study medication on days in which they did not provide responses in the medication diary), compliance rates were 63.3 percent and 54.8 percent for the multivitamin and placebo arms, respectively $(P=.21)$.

\section{Incidence of RAS episodes}

We examined the incidence of new RAS episodes in an intent-to-treat analysis of all 160 randomized participants, after adjusting for the duration of study participation. Thus, we compared the incidence in the 83 participants in the multivitamin arm (who collectively reported 348 RAS episodes) with that in the 77 participants in the placebo arm (who collectively reported 354 episodes). The results show no significant difference in the mean number of new RAS episodes between that in the multivitamin arm and that in the placebo arm during the study period (Table 2). The results of factorial analysis of variance showed no treatment effect $(P=.69)$ and no interaction between treatment and group (that is, A or B) $(P=.40)$. However, we found a significant A/B group effect $(P=.025)$. Participants with a history of more frequent RAS episodes (group B) reported, on average, almost twice as many new RAS episodes (4.79) during the study period as did participants who had a history of less frequent RAS episodes (group A, 2.50 new episodes). Sex did not interact with treatment or A/B group, and the results showed no main effect for sex.

\section{Duration of RAS episodes}

Twenty-two participants in the multivitamin arm and 14 participants in the placebo arm did not experience any new RAS episodes during the study period; therefore, we could not include them in the duration analyses. Thus, we compared the duration of episodes in 61 participants in the multivitamin arm who experienced at least one RAS episode during the study period with that in 63 participants in the placebo arm who experienced at least one RAS episode. The mean duration of each RAS episode in participants in the multivitamin arm was 8.66 days compared with 8.99 days in those in the placebo arm $(P=.60)$ (Table 3$)$. A/B group and sex did not interact significantly with treatment with regard to duration of RAS episodes, and there was no main effect for either variable.

\section{Mouth pain during RAS episodes}

We excluded from this analysis 36 of 160 participants (22 in the multivitamin arm and 14 in the placebo arm) who did not experience any new RAS episodes during the study period. Therefore, we compared mouth pain during RAS episodes in 61 participants in the multivitamin arm with that in 63 participants in the placebo arm. We found no differences between the multivitamin and placebo arms regarding worst pain $(P=.63)$, least pain $(P=$. 58), average pain $(P=.57)$ or pain right now $(P=.50)$ (Table 4). A/B group and sex did not interact significantly with treatment with regard to mouth pain scores, and there was no main effect for either variable.

\section{Normalcy of diet during RAS episodes}

Thirty-nine participants (22 in the multivitamin arm and 17 in the placebo arm) did not provide any normalcy of diet data during an RAS episode; therefore, we compared data from 61 participants in the multivitamin arm with data from 60 participants in the placebo arm for these analyses. The mean diet scores were similar (9.48 for the multivitamin arm and 9.70 for the placebo arm; $P=.18$ ). A/B group and sex did not interact significantly with treatment with regard to diet scores, and there was no main effect for either variable. 


\section{DISCUSSION}

In a subset of patients with RAS, specific replacement therapy to correct vitamin deficiencies can improve the patient's condition or induce remission. ${ }^{9}, 10,13,14$ However, with the exception of some specialists, clinicians rarely perform serum testing for vitamin deficiencies in patients with RAS. In this study, we examined the merits of an alternative approach: daily supplementation in all patients with RAS with the U.S. RDI of essential vitamins. Multivitamin supplements at these levels are safe and have been recommended for various population groups. ${ }^{21,22}$ The safety of this regimen also was evidenced by the absence of reported adverse events associated with its use in our study population.

The number of new RAS episodes did not differ significantly according to treatment arm. As expected, participants with fewer RAS episodes before enrollment experienced fewer episodes during the study period than did participants with a history of more frequent RAS episodes. These findings validate the stratification strategy used, which balanced expected numbers of RAS episodes across treatment arms.

Because the major morbidity of these ulcerative lesions results from pain, any reduction in the duration of the lesions can have significant benefits for patients. However, neither the mean duration of RAS episodes nor the associated pain was significantly different between the two arms. Normalcy of diet scores were high in both groups, indicating that minor RAS did not have a significant effect on the consistency of foods that participants could tolerate. However, because patients with RAS often report pain on consuming acidic and spicy foods, a diet scale focused on such attributes may better quantify the effects of RAS on diet than does the consistency-based scale we used.

Limitations of this study include the reliance on self-reported data from participants to determine the presence and duration of RAS episodes. However, participants received a clinical examination during study visits for almost 75 percent of all reported episodes of RAS. The dentist or dental hygienist determined that 13 percent of these episodes were not RAS, and we removed them from our analyses. If we were to extrapolate this finding to the remaining 25 percent of reported episodes for which a clinical examination was not possible, we can estimate that only an additional 3.25 percent of these episodes may not have been RAS. We would not expect this to change the outcome of the study.

Another limitation is the inherent difficulty in measuring compliance with pill taking, particularly in a yearlong trial. Thus, we cannot prove that participants took the study medication exactly as reported in their written diaries. Although some helpful devices are available, such as bottle caps that register how often the bottle is opened, the cost of these for a large and long clinical trial can be prohibitive.

Many investigators in the studies cited earlier found that high-dose replacement therapy with specific vitamins was helpful in the treatment of RAS in patients with documented vitamin deficiencies. This may explain the lack of effect in our study, in which we examined lowdose prophylactic multivitamin use in a population largely without vitamin deficiencies. In a randomized, double-masked, placebo-controlled trial of once-daily sublingual vitamin $\mathrm{B}_{12}$ therapy for RAS, Volkov and colleagues ${ }^{23}$ reported that after six months of treatment, 20 of 27 patients (74 percent) with RAS in the treatment arm were free of ulceration, compared with eight of 25 patients ( 32 percent) with RAS in the placebo arm $(P<.01)$. This response to vitamin $B_{12}$ was independent of the participant's initial blood $B_{12}$ level, which was normal in most participants. These investigators ${ }^{23}$ tested 1,000 micrograms of vitamin $\mathrm{B}_{12}$ daily compared with $6 \mu \mathrm{g}$ in our multivitamin supplement, which may account for the different outcome in our study. 
The findings of our study suggest that in the U.S. population, routine screening of all patients who have RAS for vitamin $B_{12}$ and folate deficiencies is not warranted. Although not studied here, clinicians may wish to test for such deficiencies in patients with refractory RAS or when signs suggestive of such deficiency (for example, tongue depapillation for vitamin $\mathrm{B}_{12}$ deficiency) are present.

\section{CONCLUSION}

Our study findings show that daily multivitamin supplementation, with the RDI of essential vitamins, did not result in a reduction in the number or duration of RAS episodes. Therefore, clinicians should not use this prophylactic strategy routinely in patients who have RAS.

\section{Acknowledgments}

This study was supported by The Patrick and Catherine Weldon Donaghue Medical Research Foundation, National Institutes of Health (NIH) General Clinical Research Center grant M01R006192 and NIH career development grant K23DE016946.

The authors gratefully acknowledge the contributions of those who participated in this study, and of the following people who contributed to the conduct of the clinical study: Sandra D'Amato-Palumbo, Lisa Jaser, Ruth LaCasse, Easwar Natarajan, Alis Ohlheiser, Vijay Parashar, Douglas Peterson, Margaret Phillipi and Harriet Potts.

\section{ABBREVIATION KEY}

$\begin{array}{ll}\text { RAS } & \text { Reccurent aphthous stomatitis } \\ \text { RBC } & \text { Red blood cell } \\ \text { RDI } & \text { Reference daily intake }\end{array}$

\section{References}

1. Kleinman DV, Swango PA, Pindborg JJ. Epidemiology of oral mucosal lesions in United States schoolchildren: 1986-87. Community Dent Oral Epidemiol. 1994; 22(4):243-253. [PubMed: 7924239]

2. Scully C. Clinical practice. Aphthous ulceration. N Engl J Med. 2006; 355(2):165-172. [PubMed: 16837680]

3. Silverman S Jr. Mucosal lesions in older adults. JADA. 2007; 138(suppl):41S-46S. [PubMed: 17761845]

4. Tabolli S, Bergamo F, Alessandroni L, Di Pietro C, Sampogna F, Abeni D. Quality of life and psychological problems of patients with oral mucosal disease in dermatological practice. Dermatology. 2009; 218(4):314-320. [PubMed: 19174600]

5. Hapa A, Aksoy B, Polat M, Aslan U, Atakan N. Does recurrent aphthous stomatitis affect quality of life? A prospective study with 128 patients evaluating different treatment modalities. J Dermatolog Treat. 2011; 22(4):215-220. [PubMed: 20804437]

6. Weusten BL, van de Wiel A. Aphthous ulcers and vitamin B12 deficiency. Neth J Med. 1998; 53(4): 172-175. [PubMed: 9825643]

7. Piskin S, Sayan C, Durukan N, Senol M. Serum iron, ferritin, folic acid, and vitamin B12 levels in recurrent aphthous stomatitis. J Eur Acad Dermatol Venereol. 2002; 16(1):66-67. [PubMed: 11952294]

8. Porter SR, Scully C, Flint S. Hematologic status in recurrent aphthous stomatitis compared with other oral disease. Oral Surg Oral Med Oral Pathol. 1988; 66(1):41-44. [PubMed: 3165514]

9. Wray D, Ferguson MM, Hutcheon WA, Dagg JH. Nutritional deficiencies in recurrent aphthae. J Oral Pathol. 1978; 7(6):418-423. [PubMed: 105102]

10. Wray D, Ferguson MM, Mason DK, Hutcheon AW, Dagg JH. Recurrent aphthae: treatment with vitamin B12, folic acid, and iron. Br Med J. 1975; 2(5969):490-493. [PubMed: 1148667] 
11. Thongprasom K, Youngnak P, Aneksuk V. Hematologic abnormalities in recurrent oral ulceration. Southeast Asian J Trop Med Public Health. 2002; 33(4):872-877. [PubMed: 12757242]

12. Haisraeli-Shalish M, Livneh A, Katz J, Doolman R, Sela BA. Recurrent aphthous stomatitis and thiamine deficiency. Oral Surg Oral Med Oral Pathol Oral Radiol Endod. 1996; 82(6):634-636. [PubMed: 8974135]

13. Nolan A, McIntosh WB, Allam BF, Lamey PJ. Recurrent aphthous ulceration: vitamin B1, B2 and B6 status and response to replacement therapy. J Oral Pathol Med. 1991; 20(8):389-391. [PubMed: 1941656]

14. Porter S, Flint S, Scully C, Keith O. Recurrent aphthous stomatitis: the efficacy of replacement therapy in patients with underlying hematinic deficiencies. Ann Dent. 1992; 51(2):14-16. [PubMed: 1463310]

15. Pedersen A, Hougen HP, Klausen B, Winther K. LongoVital in the prevention of recurrent aphthous ulceration. J Oral Pathol Med. 1990; 19(8):371-375. [PubMed: 2250227]

16. Pedersen A, Klausen B, Hougen HP, Ryder L, Winther K. Immunomodulation by LongoVital in patients with recurrent aphthous ulceration. J Oral Pathol Med. 1990; 19(8):376-380. [PubMed: 1979083]

17. Council for Responsible Nutrition. [Accessed March 1, 2012] Vitamin and mineral recommendations. www.crnusa.org/about_recs3.html

18. Cleeland CS, Ryan KM. Pain assessment: global use of the Brief Pain Inventory. Ann Acad Med Singapore. 1994; 23(2):129-138. [PubMed: 8080219]

19. List MA, Ritter-Sterr C, Lansky SB. A performance status scale for head and neck cancer patients. Cancer. 1990; 66(3):564-569. [PubMed: 2364368]

20. Axéll T, Henricsson V. The occurrence of recurrent aphthous ulcers in an adult Swedish population. Acta Odontol Scand. 1985; 43(2):121-125. [PubMed: 3863446]

21. Fairfield KM, Fletcher RH. Vitamins for chronic disease prevention in adults: scientific review. JAMA. 2002; 287(23):3116-3126. [PubMed: 12069675]

22. Fletcher RH, Fairfield KM. Vitamins for chronic disease prevention in adults: clinical applications. JAMA. 2002; 287(23):3127-3129. [PubMed: 12069676]

23. Volkov I, Rudoy I, Freud T, et al. Effectiveness of vitamin B12 in treating recurrent aphthous stomatitis: a randomized, double-blind, placebo-controlled trial. J Am Board Fam Med. 2009; 22(1):9-16. [PubMed: 19124628] 


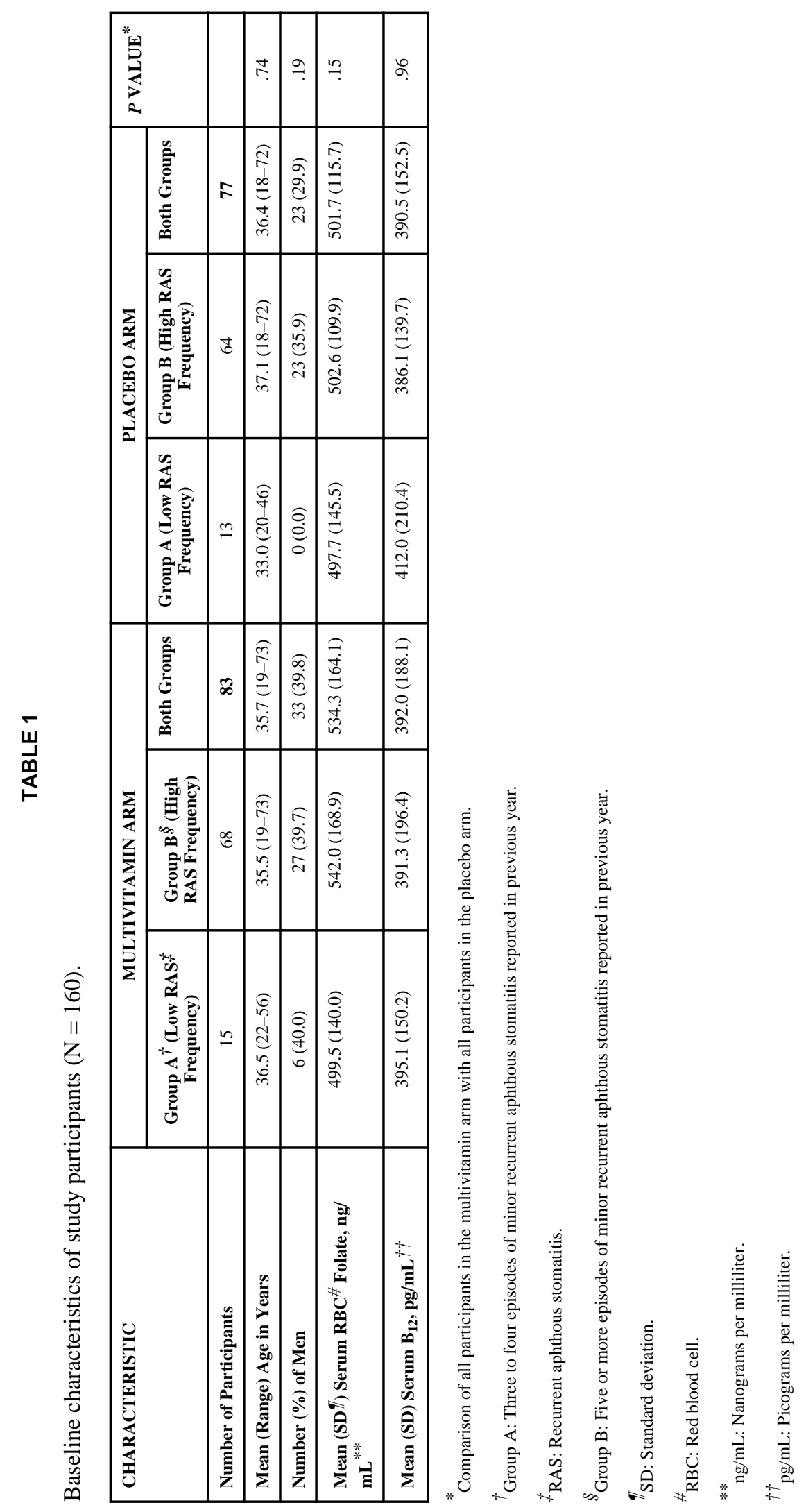


TABLE 2

Incidence of recurrent aphthous stomatitis episodes, according to treatment arm and stratification group.

\begin{tabular}{|l|c|r|}
\hline GROUP & MEAN (SD ${ }^{*}$ NUMBER OF NEW EPISODES & NUMBER OF PARTICIPANTS \\
\hline Placebo & & \\
$\mathbf{A}^{\dagger}$ & $1.92(2.99)$ & 13 \\
$\mathbf{B}^{\dagger}$ & $5.14(4.67)$ & 64 \\
TOTAL & $4.60(4.58)$ & $\mathbf{7 7}$ \\
\hline Multivitamin & & \\
A & $3.00(4.29)$ & 15 \\
B & $4.46(4.82)$ & 68 \\
TOTAL & $4.19(4.74)$ & $\mathbf{8 3}$ \\
\hline Placebo and Multivitamin Arms Combined & & \\
A & $2.50(3.72)$ & 132 \\
B & $4.79(4.75)$ & $\mathbf{1 6 0}$ \\
TOTAL & $4.39(4.65)$ & \\
\hline
\end{tabular}

* SD: Standard deviation.

${ }^{\dagger}$ Group A: Three to four episodes of minor recurrent aphthous stomatitis reported in previous year.

${ }^{*}$ Group B: Five or more episodes of minor recurrent aphthous stomatitis reported in previous year. 
TABLE 3

Duration of recurrent aphthous stomatitis (RAS) episodes.

\begin{tabular}{|l|c|c|c|}
\hline TREATMENT ARM & $\begin{array}{c}\text { MEAN (SD*) DURATION } \\
\text { IN DAYS }\end{array}$ & NUMBER OF PARTICIPANTS & NUMBER OF EPISODES \\
\hline Placebo & $8.99(5.22)$ & $63^{\dagger}$ & 329 \\
\hline Multivitamin & $8.66(4.60)$ & $61^{\dagger}$ & 335 \\
\hline $\begin{array}{l}\text { Placebo and Multivitamin Arms } \\
\text { Combined }\end{array}$ & $8.83(4.92)$ & $\mathbf{1 2 4}$ & $\mathbf{6 6 4}$ \\
\hline
\end{tabular}

* SD: Standard deviation.

${ }^{\dagger}$ Fourteen participants in the placebo arm were excluded from the duration analysis because they did not experience any new RAS episodes during study participation.

Fwenty-two participants in the multivitamin arm were excluded from the duration analysis because they did not experience any new RAS episodes during study participation. 
TABLE 4

Mouth pain scores during episodes of recurrent aphthous stomatitis (RAS).

\begin{tabular}{|l|c|c|c|c|}
\hline \multirow{2}{*}{ TREATMENT ARM } & \multicolumn{4}{|c|}{ MEAN* $^{*}\left(\right.$ SD $\left.^{\dagger}\right)$ SCORE } \\
\cline { 2 - 5 } & Worst Pain & Least Pain & Average Pain & Pain Now \\
\hline Placebo $(\mathbf{n}=\mathbf{6 3})^{\ddagger}, \S$ & $2.96(2.10)$ & $1.55(1.68)$ & $2.12(1.86)$ & $2.14(1.89)$ \\
\hline Multivitamin $(\mathbf{n}=\mathbf{6 1})^{\mathbb{I}, \#}$ & $2.83(2.19)$ & $1.42(1.64)$ & $2.07(1.89)$ & $2.03(1.93)$ \\
\hline
\end{tabular}

* Mean pain score measured on a scale from 0 to 10 .

${ }^{\dagger}$ SD: Standard deviation.

* The total number of observations in the placebo arm was 2,759 .

$\S_{\text {Fourt }}$ during study participation.

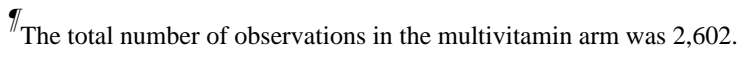

\# Twenty-two participants in the multivitamin arm were excluded from the analysis of pain scores because they did not experience any new RAS episodes during study participation. 